OPEN ACCESS

Edited by:

Wen-Chi Chou,

Chang Gung University, Taiwan

Reviewed by:

Shau-Hsuan Li,

Kaohsiung Chang Gung Memorial

Hospital, Taiwan

ChiaYen Hung,

Mackay Memorial Hospital, Taiwan

*Correspondence:

Shao-bin Chen

chensb535176@hotmail.com

Specialty section:

This article was submitted to

Surgical Oncology,

a section of the journal

Frontiers in Surgery

Received: 03 August 2021 Accepted: 30 November 2021

Published: 20 December 2021

Citation:

Chen S-b, Liu D-t and Chen Y-p (2021) The Impact of Preoperative Nutritional Status on the Survival of Patients With Esophageal Squamous Cell Carcinoma

Front. Surg. 8:752792

doi: 10.3389/fsurg.2021.752792

\section{The Impact of Preoperative} Nutritional Status on the Survival of Patients With Esophageal Squamous Cell Carcinoma

\author{
Shao-bin Chen*, Di-tian Liu and Yu-ping Chen \\ Department of Thoracic Surgery, Cancer Hospital of Shantou University Medical College, Shantou, China
}

Background: The goal of this study was to investigate the impact of different nutritional parameters in patients with esophageal squamous cell carcinoma (ESCC) who underwent surgical resection.

Methods: A total of 620 patients with ESCC who underwent esophagectomy were analyzed. A receiver operating characteristic curve was constructed to set the appropriate cutoff points for five nutritional parameters: serum albumin (SA), body mass index (BMI), geriatric nutritional risk index (GNRI), prognostic nutritional index (PNI), and a new modified nutritional risk index (mNRI). Survival analyses were performed to calculate overall survival and investigate the independent prognostic factors.

Results: The median preoperative BMI, SA, GNRI, PNI, and mNRI values were 20.90, $42.75,102.95,51.90$, and 63.90 , respectively. The corresponding optimal cutoff points were 18.75 for $\mathrm{BMl}, 43.05$ for SA, 98.5 for $\mathrm{GNRI}, 51.45$ for $\mathrm{PNI}$, and 61.45 for $\mathrm{mNRI}$. All nutritional parameters were significantly correlated with tumor length and $\mathrm{pT}$ category. Decreased nutritional parameters were significantly correlated with poor survival in univariate analysis; however, only the $\mathrm{mNRI}$ was an independent prognostic factor in multivariate analysis $(P=0.041)$.

Conclusions: Nutritional parameters are convenient and valuable prognostic factors in ESCC patients who undergo surgical resection. The new $\mathrm{mNRI}$ parameter may be superior to the other nutritional parameters.

Keywords: esophageal neoplasm, nutrition, prognosis, squamous cell carcinoma, surgery

\section{INTRODUCTION}

Esophageal carcinoma is a common digestive system malignancy. Esophagectomy remains the most important tool for treatment in resectable cases. Malnutrition is often observed in patients with digestive system malignancies, especially esophageal cancer. Almost $90 \%$ of the patients with esophageal cancer reported dysphagia as their major symptom at diagnosis, which could lead to reduced food intake and therefore impact the nutritional status of these patients (1). Moreover, esophageal cancer leads to increased energy consumption, which can also contribute to malnutrition (2).

Preoperative nutritional status has been found to be correlated with postoperative complications and outcomes in patients with malignancies $(3,4)$. Recently, various preoperative nutritional 
parameters have been identified as tumor biomarkers, such as body mass index (BMI), serum albumin (SA), geriatric nutritional risk index (GNRI), and prognostic nutritional index (PNI) (5-8). Previous studies found that these nutritional parameters might be correlated with survival in patients with esophageal squamous cell carcinoma (ESCC) (9-12). However, the results are still controversial (13), and few studies have evaluated the predictive accuracy among the different nutritional parameters in patients with ESCC who underwent esophagectomy.

In this study, we investigated the value of different nutritional parameters in patients with ESCC who underwent surgical resection and aimed to determine a parameter that is more convenient and valuable in clinical practice.

\section{PATIENTS AND METHODS}

\section{Patients}

A total of 817 patients with esophageal cancer underwent esophagectomy at Shantou University Medical College Cancer Hospital between September 2014 and December 2017. Only patients with ESCC who chose surgery as their initial treatment were included in this study. This study was approved by the Ethics Committee of our hospital and conformed to the Declaration of Helsinki. Written informed consent was signed for all patients.

\section{Pre-operative Examinations}

After the medical history taking and physical examinations, chest radiograph, barium meal, Doppler ultrasound examination of the supraclavicular lymph nodes, and contrast enhanced computed tomography scan of the chest and abdomen were routinely administrated to patients to evaluate the clinical stage of the tumor. Endoscopic ultrsonography (EUS) was also performed after the year 2010. Positron emission tomography (PET) was not routinely performed before surgery.

\section{Data Collection}

All clinicopathological data and laboratory data were obtained from the patients' medical records. The stage of the tumor was classified based on the 8th edition American Joint Committee on Cancer TNM staging system for ESCC. Weight, height, lymphocyte counts, and SA were collected within 1 week before surgery. BMI was calculated as follows: (weight, $\mathrm{kg}$ ) $/\left(\right.$ height $^{2}$, $\left.\mathrm{m}^{2}\right)$. The GNRI was calculated as $(1.489 \times \mathrm{SA}, \mathrm{g} / \mathrm{l})+(41.7 \times$ present/ideal body weight) (14). The PNI was calculated as $10 \times$ $\mathrm{SA}(\mathrm{g} / \mathrm{dL})+0.005 \times$ total lymphocyte count $\left(\right.$ per $\left.\mathrm{mm}^{3}\right)$. As the GNRI was too complicated to calculate, we tried to create a new modified nutritional risk index ( $\mathrm{mNRI}$ ) which could integrate SA and body weight in a simple pattern. As the SA and BMI have been identified as tumor biomarkers in previous studies, we set the new $\mathrm{mNRI}$ as SA $(\mathrm{g} / \mathrm{l})+\mathrm{BMI}$, and try to compared the value of this new integrated parameter to previous.

\section{Surgery}

Most of the patients underwent esophagectomy through a right thoracotomy, while other patients underwent a left thoracotomy.
For lymphadenectomy, the regional lymph nodes in the middle mediastinal, lower mediastinal, and upper abdominal regions were routinely dissected for all patients. For patients who underwent esophagectomy through a right thoracotomy, the lymph nodes around the left and right recurrent laryngeal nerves were also dissected.

\section{Statistical Analyses}

Categorical variables were compared by the $\chi^{2}$ test or Fisher's exact test. Overall survival (OS) was calculated using the Kaplan-Meier method, and the differences between survival were compared by the log-rank test. All of the clinicopathological factors and nutritional parameters which were significant in univariate analyses were simultaneously included in multivariate Cox regression analyses to identify independent prognostic factors. The receiver operating characteristic curve (ROC) was conducted to evaluate the sensitivity and specificity for the 5year OS, and the highest Youden's index was used to identify the appropriate cutoff points for BMI, SA, GNRI, PNI, and mNRI. $P<0.05$ was set as significance. All statistical analyses were conducted in SPSS 20.0 software (IBM, Armonk, New York, USA).

\section{RESULTS}

\section{Patient Characteristics}

Of the 817 patients with esophageal carcinoma who underwent esophagectomy between September 2014 and December 2017, 761 patients were diagnosed with ESCC. One hundred and sixteen patients who received neoadjuvant therapy were excluded from this study (including 94 cases of neoadjuvant chemoradiotherapy, 13 cases of neoadjuvant radiotherapy, and 9 cases of neoadjuvant chemotherapy). Twenty-five patients lacking any follow-up data were also excluded. Thus, 620 patients were enrolled for analysis in this study. There were 477 men and 143 women, and the median age was 61 years (range, 38-84 years). The mean number of lymph nodes dissected was $26.8 \pm$ 11.0 , and the median number was 26 (range, 6-74). Based on the 8th edition TNM staging system, 283 patients (45.6\%) had pN0 disease, 207 patients (33.4\%) had pN1 disease, 102 patients (16.5\%) had $\mathrm{pN} 2$ disease, and 28 patients $(4.5 \%)$ had $\mathrm{pN} 3$ disease. Radical resection was achieved in 594 patients (95.8\%), while palliative resection was performed in 26 patients $(4.2 \%)$. The postoperative morbidity rate was $8.3 \%(51 / 620)$, including 23 cases of pulmonary infection, 17 cases of anastomotic leak, and 11 cases of other complications. The hospital mortality rate was $0.5 \%(3 / 620)$.

There were 22 patients had multiple primary malignancies (including 5 patients with synchronous malignancy and 17 patients with metachronous malignancy). The most common sites for multiple primary malignancies were head and neck in 10 cases, the esophagogastric junction in five cases, the lung in three cases, the stomach in two cases, the breast in one case, and the colon in one case. A total of 172 patients receive adjuvant therapy after esophagectomy, including 31 cases of adjuvant chemoradiotherapy, 53 cases of adjuvant chemotherapy, and 88 cases of adjuvant radiotherapy. 


\section{Selection of the Optimal Cutoff Point for BMI, SA, GNRI, PNI, and mNRI}

The median preoperative BMI, SA, GNRI, PNI, and mNRI were 20.90 (range, 13.30-32.70), 42.75 (range, 32.30-53.20), 102.95 (range, 78.80-133.10), 51.90 (range, 35.50-75.20), and 63.90 (range, 49.10-89.40), respectively. We further used the ROC curve to determine the appropriate cutoff points for BMI, SA, GNRI, PNI, and mNRI. The areas under the curve (AUCs) for OS were $0.544,0.562,0.566,0.542$, and 0.567 for BMI, SA, GNRI, PNI, and mNRI, respectively. The corresponding optimal cutoff values were 18.75 for BMI, 43.05 for SA, 98.5 for GNRI, 51.45 for PNI, and 61.45 for mNRI. Furthermore, we divided the patients into two groups based on the cutoff points of each nutritional parameter as follows: low-BMI group $(\leq 18.75)$ or high-BMI group (>18.75); low-SA group $(\leq 43.05)$ or high-SA group (>43.05); low-GNRI group $(\leq 98.5)$ or high-GNRI group (>98.5); low-PNI group $(\leq 51.45)$ or high-PNI group $(>51.45)$; and low-mNRI group $(\leq 61.45)$ or high-mNRI group $(>61.45)$.

\section{Correlation Between Nutritional Parameters and Clinicopathological Factors}

Tables 1, 2 show patient clinicopathological factors stratified by different nutritional parameters. All nutritional parameters were significantly correlated with tumor length and pT category $(P<0.05)$. A longer tumor length and advanced $\mathrm{pT}$ category were more often found in the decreased nutritional parameter groups. Moreover, patients older than 60 years were more likely to have low SA, low GNRI, low PNI, or low mNRI, while male patients were more likely to have low GNRI and low mNRI. Furthermore, palliative resection was more often performed in patients with low BMI, low SA, low GNRI, or low mNRI. However, no correlations were found between the five nutritional parameters and tumor location, histologic grade, thoracotomy, or pN category.

For postoperative complications, only SA and PNI were found to have significant correlations. Postoperative complications were more often found in patients with low SA or Low PNI. Although postoperative complications were more often found in patients with low BMI, low GNRI, or low mNRI, the differences were not significant $(P>0.05)$.

\section{Survival and Prognostic Factors}

The last follow-up was conducted in December 2020, with a mean follow-up time of 34.7 months (range, 1-69 months). Two hundred and fifteen patients died, and 10 patients were lost to follow-up (1.6\%).

The 1-, 3-, and 5-year OS rates for all patients were 88.5, 66.0 , and $61.3 \%$, respectively. The correlations between the clinicopathological factors and survival are shown in Table 3. In univariate analysis, the variables correlated with survival were tumor length, thoracotomy, resection margin, pT category, and $\mathrm{pN}$ category. Patients with a tumor length $\leq 5 \mathrm{~cm}$ had a significantly improved 5-year OS than those with a tumor length $>5 \mathrm{~cm}(66.2$ vs. $50.0 \%, P<0.001)$. Patients who underwent a left thoracotomy had a worse 5-year OS than those who underwent
TABLE 1 | Correlation of the body mass index and serum albumin with the clinicopathological features.

\begin{tabular}{|c|c|c|c|c|c|c|c|}
\hline & \multirow{2}{*}{$\begin{array}{c}\text { No. } \\
\text { patients }\end{array}$} & \multicolumn{3}{|c|}{ BMI } & \multicolumn{3}{|c|}{ SA } \\
\hline & & $\leq 18.75$ & $>18.75$ & $P$-value & $\leq 43.05$ & $>43.05$ & $P$-value \\
\hline Gender & & & & 0.086 & & & 0.444 \\
\hline Male & 477 & 113 & 364 & & 265 & 212 & \\
\hline Female & 143 & 24 & 119 & & 74 & 69 & \\
\hline Age (yr) & & & & 0.208 & & & $<0.001$ \\
\hline$\leq 60$ & 303 & 60 & 243 & & 138 & 165 & \\
\hline$>60$ & 317 & 77 & 240 & & 201 & 116 & \\
\hline $\begin{array}{l}\text { Tumor } \\
\text { location }\end{array}$ & & & & 0.622 & & & 0.206 \\
\hline Upper third & 110 & 28 & 82 & & 54 & 56 & \\
\hline Middle third & 388 & 84 & 304 & & 211 & 177 & \\
\hline Lower third & 122 & 25 & 97 & & 74 & 48 & \\
\hline Tumor length & & & & 0.044 & & & 0.022 \\
\hline$\leq 5 \mathrm{~cm}$ & 434 & 86 & 348 & & 224 & 210 & \\
\hline$>5 \mathrm{~cm}$ & 186 & 51 & 135 & & 115 & 71 & \\
\hline $\begin{array}{l}\text { Histologic } \\
\text { grade }\end{array}$ & & & & 0.164 & & & 0.287 \\
\hline Well & 210 & 52 & 158 & & 106 & 104 & \\
\hline Moderate & 322 & 72 & 250 & & 181 & 141 & \\
\hline Poor & 88 & 13 & 75 & & 52 & 36 & \\
\hline Thoracotomy & & & & 0.913 & & & 0.520 \\
\hline Left & 161 & 36 & 125 & & 92 & 69 & \\
\hline Right & 459 & 101 & 358 & & 247 & 212 & \\
\hline $\begin{array}{l}\text { Resection } \\
\text { margin }\end{array}$ & & & & 0.026 & & & 0.012 \\
\hline Radical & 594 & 126 & 468 & & 331 & 263 & \\
\hline Palliative & 26 & 11 & 15 & & 8 & 18 & \\
\hline pT category & & & & 0.039 & & & 0.026 \\
\hline $\mathrm{pT} 1$ & 73 & 12 & 61 & & 33 & 40 & \\
\hline pT2 & 102 & 21 & 81 & & 50 & 52 & \\
\hline pT3 & 373 & 79 & 294 & & 207 & 166 & \\
\hline pT4 & 72 & 25 & 47 & & 49 & 23 & \\
\hline pN category & & & & 0.975 & & & 0.332 \\
\hline pNO & 283 & 61 & 222 & & 149 & 134 & \\
\hline pN1 & 207 & 48 & 159 & & 117 & 90 & \\
\hline pN2 & 102 & 22 & 80 & & 61 & 41 & \\
\hline pN3 & 28 & 6 & 22 & & 12 & 16 & \\
\hline $\begin{array}{l}\text { Post- } \\
\text { operative } \\
\text { complications }\end{array}$ & & & & 0.336 & & & 0.017 \\
\hline Yes & 51 & 14 & 37 & & 36 & 15 & \\
\hline No & 569 & 123 & 446 & & 303 & 266 & \\
\hline $\begin{array}{l}\text { Adjuvant } \\
\text { therapy }\end{array}$ & & & & 0.664 & & & 0.712 \\
\hline Yes & 172 & 36 & 136 & & 92 & 80 & \\
\hline No & 448 & 101 & 347 & & 247 & 201 & \\
\hline
\end{tabular}

BMI, body mass index; $S A$, serum albumin.

a right thoracotomy (53.7 vs. $64.0 \%, P=0.005$ ). Moreover, patients who had advanced pT category, advanced pN category, or received palliative surgery also had significantly worse survival $(P<0.001)$. 
TABLE 2 | Correlation of the GNRI, PNI, and mNRI with the clinicopathological features.

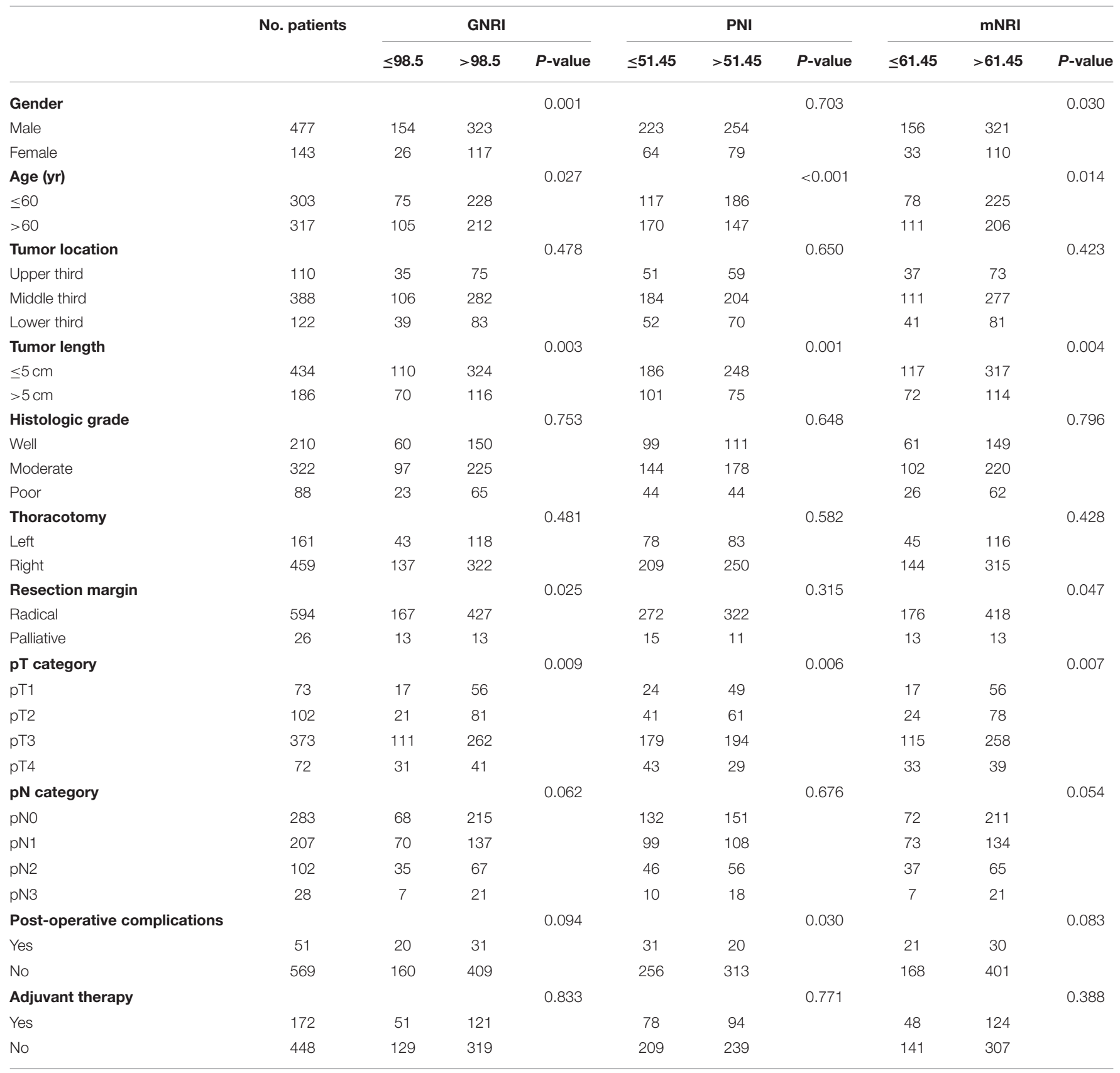

GNRI, geriatric nutritional risk index; PNI, prognostic nutritional Index; $m N R I$, modified nutritional risk index.

Table 4 shows the impact of the nutritional parameters on survival in univariate analysis. All five nutritional parameters were significantly correlated with survival (Figure 1). Patients in the decreased nutritional parameter groups had significantly worse survival than those in the higher groups.

The multivariate analysis incorporated clinicopathological factors and nutritional parameters that were significant in the univariate analyses. Thoracotomy, resection margin, $\mathrm{pT}$ category, and $\mathrm{pN}$ category were independent prognostic factors in this study; however, tumor length was not an independent risk factor $(P=0.497)$. Although all five nutritional parameters were significantly correlated with survival in univariate analyses, only the mNRI value was an independent risk factor in multivariate analysis $(P=0.041)$.

\section{DISCUSSION}

The identification of factors associated with high-risk, prior to treatment, is important for determining an individual therapeutic strategy for patients with malignancies. Currently, 
TABLE 3 | Univariate analysis and multivariate analysis in regard to overall survival according to clinicopathological factors.

\begin{tabular}{|c|c|c|c|c|}
\hline \multirow[t]{2}{*}{ Variable } & \multicolumn{2}{|c|}{ Univariate analysis } & \multicolumn{2}{|c|}{ Multivariate analysis } \\
\hline & 5-yr OS (\%) & $P$-value & HR $(95 \% \mathrm{Cl})$ & $P$-value \\
\hline Gender & & 0.129 & & \\
\hline Male & 59.4 & & & \\
\hline Female & 67.6 & & & \\
\hline Age (yr) & & 0.925 & & \\
\hline$\leq 60$ & 61.1 & & & \\
\hline$>60$ & 61.6 & & & \\
\hline Tumor location & & 0.477 & & \\
\hline Upper third & 60.5 & & & \\
\hline Middle third & 62.5 & & & \\
\hline Lower third & 56.7 & & & \\
\hline Tumor length & & $<0.001$ & 1.107 (0.826-1.484) & 0.497 \\
\hline$\leq 5 \mathrm{~cm}$ & 66.2 & & & \\
\hline$>5 \mathrm{~cm}$ & 50.0 & & & \\
\hline Histologic grade & & 0.289 & & \\
\hline Well & 64.4 & & & \\
\hline Moderate & 59.0 & & & \\
\hline Poor & 63.4 & & & \\
\hline Thoracotomy & & 0.005 & $0.579(0.433-0.774)$ & $<0.001$ \\
\hline Left thoracotomy & 53.7 & & & \\
\hline Right thoracotomy & 64.0 & & & \\
\hline Resection margin & & $<0.001$ & $2.923(1.707-5.005)$ & $<0.001$ \\
\hline Radical & 62.9 & & & \\
\hline Palliative & 21.8 & & & \\
\hline pT category & & $<0.001$ & 1.356 (1.084-1.695) & 0.008 \\
\hline pT1 & 88.2 & & & \\
\hline pT2 & 63.6 & & & \\
\hline рT3 & 61.1 & & & \\
\hline pT4 & 33.2 & & & \\
\hline pN category & & $<0.001$ & $1.790(1.548-2.070)$ & $<0.001$ \\
\hline pNO & 75.8 & & & \\
\hline pN1 & 58.1 & & & \\
\hline pN2 & 39.4 & & & \\
\hline pN3 & 21.2 & & & \\
\hline $\begin{array}{l}\text { Post-operative } \\
\text { complications }\end{array}$ & & 0.378 & & \\
\hline Yes & 58.0 & & & \\
\hline No & 62.9 & & & \\
\hline Adjuvant therapy & & 0.235 & & \\
\hline Yes & 63.2 & & & \\
\hline No & 60.4 & & & \\
\hline
\end{tabular}

Cl, confidence interval; HR, hazard ratio; MST, median survival time; OS, overall survival.

the TNM staging system is widely used for predicting the outcomes of esophageal cancer and other malignancies. Although a separate clinical stage (cTNM) was provided in the 8th edition for ESCC to be used as a prognostic indicator before treatment, its predictive value is still limited. The clinical stage is mainly determined by imaging, so it may be limited by individual techniques (15). The Worldwide Esophageal Cancer Collaboration (WECC) recommended
TABLE 4 | Univariate analysis and multivariate analysis in regard to overall survival according to nutritional parameters.

\begin{tabular}{|c|c|c|c|c|}
\hline \multirow[t]{2}{*}{ Variable } & \multicolumn{2}{|c|}{ Univariate analysis } & \multicolumn{2}{|c|}{ Multivariate analysis } \\
\hline & 5-yr OS (\%) & $P$-value & HR (95\% Cl) & $P$-value \\
\hline SA & & 0.011 & $0.894(0.618-1.294)$ & 0.554 \\
\hline$\leq 43.05$ & 56.8 & & & \\
\hline$>43.05$ & 67.6 & & & \\
\hline BMI & & 0.045 & $0.854(0.590-1.235)$ & 0.401 \\
\hline$\leq 18.75$ & 53.3 & & & \\
\hline$>18.75$ & 63.5 & & & \\
\hline mNRI & & $<0.001$ & $0.478(0.216-0.986)$ & 0.041 \\
\hline$\leq 61.45$ & 49.8 & & & \\
\hline$>61.45$ & 66.7 & & & \\
\hline PNI & & 0.038 & $0.973(0.696-1.359)$ & 0.872 \\
\hline$\leq 51.45$ & 56.0 & & & \\
\hline$>51.45$ & 65.9 & & & \\
\hline GNRI & & 0.001 & $1.594(0.708-3.591)$ & 0.261 \\
\hline$\leq 98.5$ & 51.5 & & & \\
\hline$>98.5$ & 65.6 & & & \\
\hline
\end{tabular}

BMI, body mass index; $\mathrm{Cl}$, confidence interval; GNRI, geriatric nutritional risk index; $H R$, hazard ratio; $m N R I$, modified nutritional risk index; MST, median survival time; OS, overall survival; PNI, prognostic nutritional Index; SA, serum albumin.

that a series of examinations be conducted to obtain a reliable cTNM stage, but not all of these modalities were available in every institution (15). Therefore, we think that it is necessary to develop other easily accessible and effective indicators to predict the outcome of esophageal cancer patients before treatment, which may help to improve individualized treatment.

Preoperative malnutrition has been reported to be a predictor of postoperative complications and outcomes in cancer patients and can be used as a prognostic indicator (16-18). Most nutritional parameters, such as ALB and BMI, can be easily obtained in clinical examinations and routine laboratory examinations. However, these parameters may be easily affected by many factors, such as inflammation or hydration status (1921). Recently, other nutritional parameters, such as the GNRI and PNI, have been proposed to evaluate nutritional-related risk. Previous studies also found that these nutritional parameters were independent prognostic factors in different kinds of cancers $(3,8,22,23)$. However, to date, few studies have compared the prognostic accuracy of different nutritional parameters in ESCC patients.

In this study, we investigated the value of different nutritional parameters, including BMI, SA, GNRI, PNI, and a new mNRI, in ESCC patients who underwent surgical resection. We found that all nutritional parameters were significantly correlated with tumor length and pT category. A longer tumor length and advanced pT category were more often found in the decreased nutritional parameter groups. It is easy to understand that the nutritional condition for patients with esophageal cancer is correlated with tumor length and invasion depth, as patients with larger primary tumor sizes may suffer a longer period 

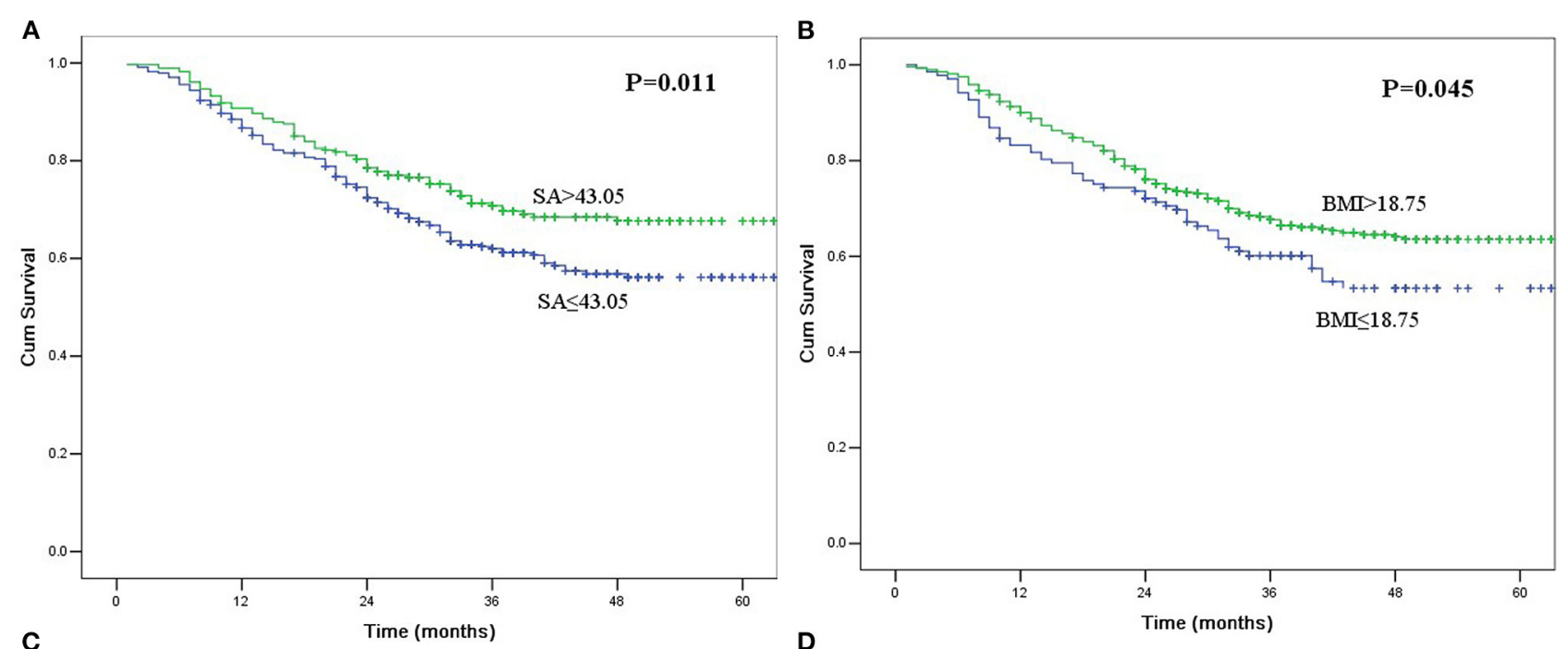

C

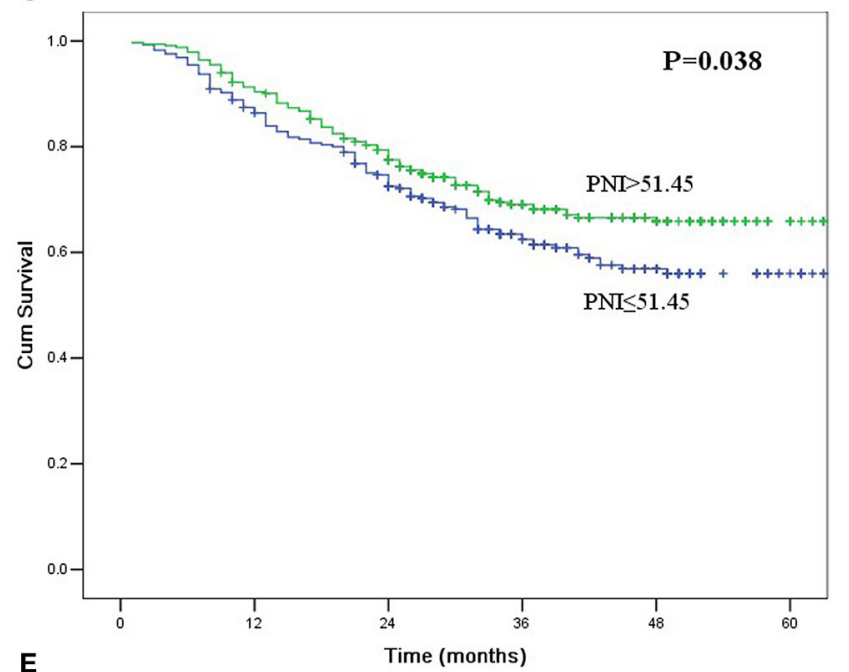

E
D

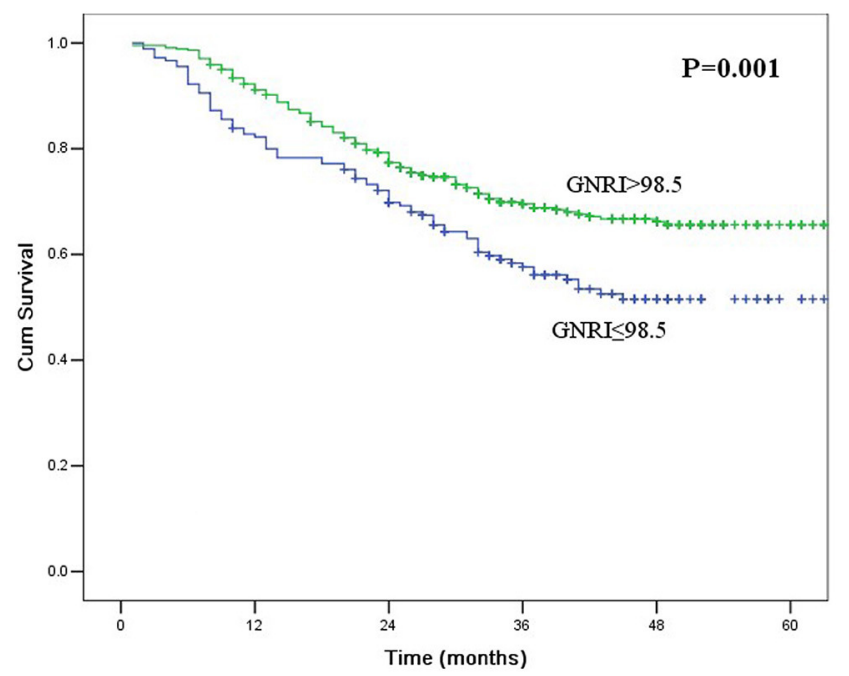

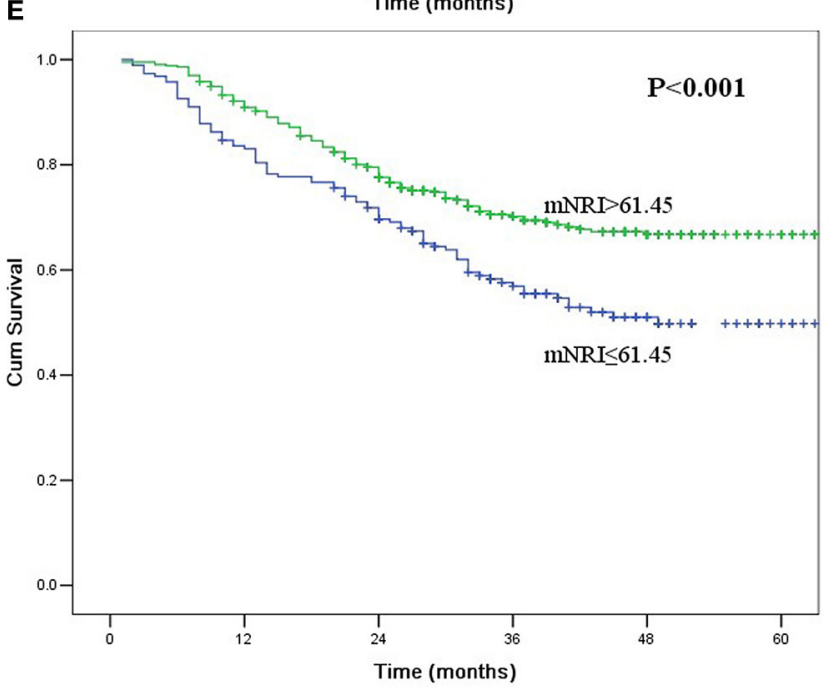

FIGURE 1 | Kaplan-Meier curves for overall survival according to serum albumin (SA, A), body mass index (BMI, B), prognostic nutritional index (PNI, C), geriatric nutritional risk index (GNRI, D), and modified nutritional risk index (mNRI, E). All of the survival differences were significant $(P<0.05)$. 
of dysphagia and more serious symptoms, which may lead to insufficient oral intake and malnutrition.

Although all five nutritional parameters were significantly correlated with survival in univariate analyses, only the mNRI was an independent risk factor in multivariate analyses in our study. Moreover, the AUC for OS of the mNRI was higher than that of the other nutritional parameters. These results indicate that the new nutritional parameter (mNRI) could be used as an indicator to evaluate the prognosis of ESCC patients who underwent surgery and might be more effective than the other nutritional parameters. Further studies are needed to elucidate the correlation between our new nutritional parameter (mNRI), other nutritional parameters and the prognosis of patients with ESCC.

The mechanism by which malnutrition correlates with the poor prognosis of cancer patients is still not clear. There are several possible explanations. First, malnutrition may be associated with immune suppression in patients, which may provide a favorable microenvironment for tumor recurrence and lead to cancer recurrence after surgery $(24,25)$. Second, the presence of malnutrition might decrease the tolerance and response to treatment, which might also lead to the poor prognosis of cancer patients. Andreyev et al. (26) found that the poor survival of gastrointestinal cancer patients with malnutrition undergoing chemotherapy might be a result of treatment with a lower dose. Di Fiore et al. (27) found that for patients with esophageal cancer who underwent chemoradiotherapy, a higher SA level was a predictor of a complete response. Third, preoperative malnutrition may increase postoperative complications and the mortality rate. The esophagectomy procedure is very invasive with a higher mortality rate than other gastrointestinal cancers, while pulmonary complications are one of the most important factors for perioperative mortality (28). Previous studies have found that malnutrition patients who undergo esophagectomy may develop more pulmonary complications. Kamachi et al. (29) analyzed 340 esophageal cancer patients who underwent esophagectomies and found that malnutrition increased postoperative pulmonary complications. Masoomi et al. (17) analyzed 6,352 esophageal cancer patients who underwent esophagectomies and found that weight loss was the most important factor for acute respiratory failure. Finally, malnutrition patients may have a higher risk of non-cancer death. Dignam et al. (30) found that in breast cancer, underweight patients suffered a higher rate of non-cancer death than normal-weight patients. Migita et al. (31) found that more underweight patients with gastric cancer died of noncancer causes, especially infection, than normal-weight patients

\section{REFERENCES}

1. Djarv T, Lagergren P. Quality of life after esophagectomy for cancer. Expert Rev Gastroenterol Hepatol. (2012) 6:115-22. doi: 10.1586/eg h.11.91

2. Martin L, Lagergren P. Long-term weight change after oesophageal cancer surgery. Br J Surg. (2009) 96:1308-14. doi: 10.1002/bjs.6723 with gastric cancer. According to these theories, we think that a perioperative nutritional intervention, such as administration of immunonutrition and dietary counseling, may improved the nutritional status and tolerance to treatment in malnutrition patients with malignancies. However, more studies are needed to further elucidate the mechanism and evaluate our hypothesis.

The major limitation of this study was that it was retrospective and single-center in nature. Moreover, the AUC for OS for all five nutritional parameters was small, indicating that the diagnostic accuracy of these parameters was low. We think that multicenter studies with larger cohorts are needed to evaluate our findings in this study, especially the value of our new mNRI for patients with ESCC.

In conclusion, our study demonstrated that nutritional parameters were convenient and valuable prognostic predictors in patients with ESCC who underwent surgical resection. A new mNRI parameter might be superior to the other nutritional parameters in prognosis evaluation.

\section{DATA AVAILABILITY STATEMENT}

The raw data supporting the conclusions of this article will be made available by the authors, without undue reservation.

\section{ETHICS STATEMENT}

The studies involving human participants were reviewed and approved by Ethics Committee of Shantou University Medical College Cancer Hospital. The patients/participants provided their written informed consent to participate in this study.

\section{AUTHOR CONTRIBUTIONS}

S-bC designed the research, analyzed the data, and wrote part of the paper. D-tL analyzed the data and wrote part of the paper. Y-pC designed the research and analyzed the data. All authors contributed to the article and approved the submitted version.

\section{FUNDING}

This work was supported by the Medical Scientific Research Foundation of Guangdong Province of China (B2019070).

\section{ACKNOWLEDGMENTS}

We would like to thank American Journal Experts (www.aje.cn) for English language editing. Stanga Z. Nutritional risk is a clinical predictor of postoperative mortality and morbidity in surgery for colorectal cancer. Br J Surg. (2010) 97:92-7. doi: 10.1002/bjs.6805

4. Caccialanza R, Pedrazzoli P, Cereda E, Gavazzi C, Pinto C, Paccagnella A, et al. Nutritional support in cancer patients: a position paper from the Italian Society of Medical Oncology (AIOM) and the Italian Society 
of Artificial Nutrition and Metabolism (SINPE). J Cancer. (2016) 7:131-5. doi: 10.7150/jca. 13818

5. Bickenbach KA, Denton B, Gonen M, Brennan MF, Coit DG, Strong VE. Impact of obesity on perioperative complications and long-term survival of patients with gastric cancer. Ann Surg Oncol. (2013) 20:780-7. doi: 10.1245/s10434-012-2653-3

6. Kanda M, Fujii T, Kodera Y, Nagai S, Takeda S, Nakao A. Nutritional predictors of postoperative outcome in pancreatic cancer. Br J Surg. (2011) 98:268-74. doi: 10.1002/bjs.7305

7. Nassri A, Zhu H, Wang DH, Ramzan Z. Serum albumin at diagnosis is an independent predictor of early mortality in veteran patients with esophageal cancer. Nutr Cancer. (2018) 70:1246-53. doi: 10.1080/01635581.2018.1512639

8. Miyake $\mathrm{H}$, Tei $\mathrm{H}$, Fujisawa $M$. Geriatric nutrition risk index is an important predictor of cancer-specific survival, but not recurrence-free survival, in patients undergoing surgical resection for non-metastatic renal cell carcinoma. Curr Urol. (2017) 10:26-31. doi: 10.1159/000447147

9. Wu N, Chen G, Hu H, Pang L, Chen Z. Low pretherapeutic serum albumin as a risk factor for poor outcome in esophageal squamous cell carcinomas. Nutr Cancer. (2015) 67:481-5. doi: 10.1080/01635581.2015.1004726

10. Wang P, Li Y, Sun H, Liu S, Zhang R, Liu X, et al. Predictive value of body mass index for short-term outcomes of patients with esophageal cancer after esophagectomy: a meta-analysis. Ann Surg Oncol. (2019) 26:2090-103. doi: 10.1245/s10434-019-07331-w

11. Hirahara N, Tajima Y, Fujii Y, Kaji S, Yamamoto T, Hyakudomi R, et al. Preoperative prognostic nutritional index predicts long-term surgical outcomes in patients with esophageal squamous cell carcinoma. World J Surg. (2018) 42:2199-208. doi: 10.1007/s00268-017-4437-1

12. Migita K, Matsumoto S, Wakatsuki K, Ito M, Kunishige T, Nakade H, et al. The prognostic significance of the geriatric nutritional risk index in patients with esophageal squamous cell carcinoma. Nutr Cancer. (2018) 70:1237-45. doi: 10.1080/01635581.2018.1512640

13. Deng HY, Alai G, Li G, Luo J, Zhuo ZG, Lin YD. High BMI has no impact on the survival of Chinese patients with lower thoracic esophageal adenocarcinoma treated with curative esophagectomy: a propensity scorematched study. Dis Esophagus. (2019) 32. doi: 10.1093/dote/doy059

14. Yamana I, Takeno S, Shimaoka H, Yamashita K, Yamada T, Shiwaku H, et al. Geriatric Nutritional Risk Index as a prognostic factor in patients with esophageal squamous cell carcinoma -retrospective cohort study. Int $J$ Surg. (2018) 56:44-8. doi: 10.1016/j.ijsu.2018.03.052

15. Rice TW, Ishwaran H, Blackstone EH, Hofstetter WL, Kelsen DP, AppersonHansen C, et al. Recommendations for clinical staging (cTNM) of cancer of the esophagus and esophagogastric junction for the 8th edition AJCC/UICC staging manuals. Dis Esophagus. (2016) 29:913-9. doi: 10.1111/dote.12540

16. Mullen JT, Davenport DL, Hutter MM, Hosokawa PW, Henderson WG, Khuri $\mathrm{SF}$, et al. Impact of body mass index on perioperative outcomes in patients undergoing major intra-abdominal cancer surgery. Ann Surg Oncol. (2008) 15:2164-72. doi: 10.1245/s10434-008-9990-2

17. Masoomi H, Nguyen B, Smith BR, Stamos MJ, Nguyen NT. Predictive factors of acute respiratory failure in esophagectomy for esophageal malignancy. Am Surg. (2012) 78:1024-8. doi: 10.1177/000313481207801002

18. Pausch T, Hartwig W, Hinz U, Swolana T, Bundy BD, Hackert T, et al. Cachexia but not obesity worsens the postoperative outcome after pancreatoduodenectomy in pancreatic cancer. Surgery. (2012) 152:S81-88. doi: 10.1016/j.surg.2012.05.028

19. Wang AY, Sea MM, Ho ZS, Lui SF Li PK, Woo J. Evaluation of handgrip strength as a nutritional marker and prognostic indicator in peritoneal dialysis patients. Am J Clin Nutr. (2005) 81:79-86. doi: 10.1093/ajcn/81.1.79

20. Omran ML, Morley JE. Assessment of protein energy malnutrition in older persons, part I: history, examination, body composition, and screening tools. Nutrition. (2000) 16:50-63. doi: 10.1016/S0899-9007(99)00224-5
21. Omran ML, Morley JE. Assessment of protein energy malnutrition in older persons, Part II: laboratory evaluation. Nutrition. (2000) 16:131-40. doi: 10.1016/S0899-9007(99)00251-8

22. Bouillanne O, Morineau G, Dupont C, Coulombel I, Vincent JP, Nicolis I, et al. Geriatric nutritional risk index: a new index for evaluating at-risk elderly medical patients. Am J Clin Nutr. (2005) 82:777-83. doi: 10.1093/ajcn/82.4.777

23. Nozoe T, Kohno M, Iguchi T, Mori E, Maeda T, Matsukuma A, et al. The prognostic nutritional index can be a prognostic indicator in colorectal carcinoma. Surg Today. (2012) 42:532-5. doi: 10.1007/s00595-011-0061-0

24. Reynolds JV, Shou JA, Sigal R, Ziegler M, Daly JM. The influence of protein malnutrition on $\mathrm{T}$ cell, natural killer cell, and lymphokineactivated killer cell function, and on biological responsiveness to high-dose interleukin-2. Cell Immunol. (1990) 128:569-77. doi: 10.1016/0008-8749(90)9 0049-W

25. Good RA, West A, Day NK, Dong ZW, Fernandes G. Effects of undernutrition of host cell and organ function. Cancer Res. (1982) 42:737s-46s.

26. Andreyev HJ, Norman AR, Oates J, Cunningham D. Why do patients with weight loss have a worse outcome when undergoing chemotherapy for gastrointestinal malignancies? Eur J Cancer. (1998) 34:503-9. doi: 10.1016/S0959-8049(97)10090-9

27. Di Fiore F, Lecleire S, Pop D, Rigal O, Hamidou H, Paillot B, et al. Baseline nutritional status is predictive of response to treatment and survival in patients treated by definitive chemoradiotherapy for a locally advanced esophageal cancer. Am J Gastroenterol. (2007) 102:2557-2263. doi: 10.1111/j.1572-0241.2007.01437.x

28. Dhungel B, Diggs BS, Hunter JG, Sheppard BC, Vetto JT, Dolan JP. Patient and peri-operative predictors of morbidity and mortality after esophagectomy: American College of Surgeons National Surgical Quality Improvement Program (ACS-NSQIP), 2005-2008. J Gastrointest Surg. (2010) 14:1492-501. doi: 10.1007/s11605-010-1328-2

29. Kamachi K, Ozawa S, Hayashi T, Kazuno A, Ito E, Makuuchi H. Impact of body mass index on postoperative complications and long-term survival in patients with esophageal squamous cell cancer. Dis Esophagus. (2016) 29:229-35. doi: 10.1111/dote.12327

30. Dignam JJ, Wieand K, Johnson KA, Fisher B, Xu L, Mamounas EP. Obesity, tamoxifen use, and outcomes in women with estrogen receptorpositive early-stage breast cancer. J Natl Cancer Inst. (2003) 95:1467-76. doi: 10.1093/jnci/djg060

31. Migita K, Takayama T, Matsumoto S, Wakatsuki K, Tanaka T, Ito $\mathrm{M}$, et al. Impact of being underweight on the long-term outcomes of patients with gastric cancer. Gastric Cancer. (2016) 19:735-43. doi: $10.1007 /$ s10120-015-0531-y

Conflict of Interest: The authors declare that the research was conducted in the absence of any commercial or financial relationships that could be construed as a potential conflict of interest.

Publisher's Note: All claims expressed in this article are solely those of the authors and do not necessarily represent those of their affiliated organizations, or those of the publisher, the editors and the reviewers. Any product that may be evaluated in this article, or claim that may be made by its manufacturer, is not guaranteed or endorsed by the publisher.

Copyright $\odot 2021$ Chen, Liu and Chen. This is an open-access article distributed under the terms of the Creative Commons Attribution License (CC BY). The use, distribution or reproduction in other forums is permitted, provided the original author(s) and the copyright owner(s) are credited and that the original publication in this journal is cited, in accordance with accepted academic practice. No use, distribution or reproduction is permitted which does not comply with these terms. 